\title{
Talcott Parsons' Theorie der symbolisch generalisierten Medien in ihrem Verhältnis zu Sprache und Kommunikation *
}

\section{Jan Künzler}

Institut für Soziologie, Lehrstuhl für Soziologie II, Universität Würzburg, Wittelsbacherplatz 1, D-8700 Würzburg

Zusammenfassung: Talcott Parsons' Version einer Theorie symbolisch generalisierter Medien soll hier rekonstruiert werden, indem die Rolle der Medien als integrativer Mechanismen innerhalb seiner Theorie systemischer Differenzierung untersucht wird. Da Medien durch die ihnen zugedachte Aufgabe, sowohl Interaktion als auch Austausch zu vermitteln, überfordert werden, bleibt die Stimmigkeit der Theorie prekär: Die Konkurrenz von Geldmodell, das den Austauschaspekt, und Sprachmodell, das den Interaktionsaspekt erfassen soll, scheint zwar durch Einordnung des Geldmediums in das Sprachmodell gelöst; de facto findet aber eine monetäre Umdeutung der Sprache statt. Bei der Anwendung auf konkrete Vorgänge im Gesellschaftssystem scheitert jedoch auch die Geldanalogie. Für die Defizite der Theorie scheint das von Parsons nicht ausreichend geklärte Verhältnis zwischen Medien und Sprache verantwortlich zu sein.

Innerhalb der funktionalistischen Systemtheorie, wie sie in den USA durch Talcott Parsons und in der BRD durch Niklas Luhmann repräsentiert wird, spielt seit Mitte der fünfziger Jahre, genauer: seit Parsons' gemeinsam mit Neil Joseph Smelser verfaßter Studie ,Economy and Society (1956), die Konzeption einer Theorie symbolisch generalisierter Medien des Austausches, oder auch: der Interaktion (bei Luhmann: Medien der Kommunikation) eine zunehmend wichtige Rolle. Nach seiner Wendung zur Systemtheorie und der Einführung des AGIL-Schemas als der Grundlage einer Theorie systemischer Differenzierung sah Parsons sich gezwungen, die Existenz integrativer Mechanismen anzunehmen, die die Einheit des Differenzierten gewährleisten. In Interpenetration und den symbolisch generalisierten Medien glaubte er, derartige Mechanismen gefunden zu haben. Er hat jedoch versäumt, den mikrosoziologischen Interaktionsaspekt und den makrosoziologischen Austauschaspekt der intersystemischen Prozesse zueinander in ein definitives Verhältnis zu setzen. Diese Dichotomie wiederholt sich auf der Ebene der Medientheorie, deren Begrifflichkeit, das heißt die Bestimmung der Medieneigenschaften und -funktionen, anhand zweier Modelle, Geld und Sprache, konstruiert wird, die sich jeweils einem der beiden Aspekte zuordnen lassen: das Geldmodell dient der Erfassung des Austauschaspekts, das Sprachmodell der Erfassung des Interaktionsaspekts.

\footnotetext{
* Für ihre Unterstützung und Anteilnahme danke ich
} Franz-Xaver Kaufmann und Hans-Joachim Schulze.
Es ist die These dieser Arbeit, daß die beiden paradigmatischen Modelle der Medientheorie Parsons', Geld und Sprache, zwar scheinbar in eine einheitliche Konzeption integriert werden können, de facto jedoch inkompatibel sind, und, insofern sie in einem Verhältnis geheimer Konkurrenz stehen, die Ambiguitäten der Medientheorie zu verantworten haben. Schließlich wird die Konkurrenz allerdings stillschweigend und entgegen Parsons' expliziten Erklärungen zugunsten des Geldmodells entschieden: das Sprachmodell wird monetär umgedeutet.

Auch in Jürgen Habermas' Kritik der Medientheorie Parsons' (1981a; 1981b; 1981c) spielt das Verhältnis von Sprache und Medien eine zentrale Rolle. Habermas' Rekonstruktion verdankt sich jedoch einer spezifischen Intention, die Parsons' Grundlagen nicht unangetastet läßt. Während Parsons' Einführung der generalisierten Medien sich aus den differenzierungstheoretischen Konsequenzen des AGIL-Schemas ergeben hatte (s. u.), sind die Revisionen, die Habermas innerhalb der Medientheorie vornimmt, Folge einer Theoriekonstruktion, die Handlungstheorie und Systemtheorie, System und Lebenswelt in den Rahmen einer umfassenden kritischen Gesellschaftstheorie integrieren will. Habermas dient die Medientheorie als analytische Brücke zwischen den beiden inkommensurablen' Einzeltheorien, deren Widerspruch sich so als Schein erweist; die Phänomene medienvermittelter Interaktion sind die Klammer, die das ausdifferenzierte System materieller Reproduktion (Wirtschaft und Staat) ob der Notwendigkeit ihrer lebensweltlichen Institutionalisierung auch normativ an die Lebenswelt rückbinden (vgl. 1981c: 230). 
Dem Dualismus von System und Lebenswelt korrespondiert in Habermas' Version der Medientheorie ein Mediendualismus (vgl. 1981c: 419); Habermas legt den Schnitt zwischen Geld und Macht auf der einen, Einfluß und Wertbindung auf der anderen Seite. Obwohl er die Defizite schon einer monetären Machttheorie sieht (vgl. 1981b: 87f.), kann er doch aus theorieimmanenten Gründen nicht auf Macht als geldanaloges Medium verzichten. Habermas vernachlässigt die spezifische Differenz zwischen Sprachmodell und Geldmodell: er kann den makrosoziologisch zu erfassenden intersystemischen Austausch von Produkten und Faktoren ignorieren, da er ,System“ als Bereich zweckrationalen Handelns, also mikrosoziologisch definiert (vgl. 1981c: 231).

\section{Parsons' differenzierungstheoretische Grundlegung}

Das Konzept der symbolisch generalisierten Medien verdankt seine charakteristischen Züge dem Umstand, daß Parsons' struktur-funktionalistische Theorie' ${ }^{1}$ wesentlich eine Theorie der Differenzierung ist - zumindest seit Parsons Handlung mit systemtheoretischen Mitteln $\mathrm{zu}$ konstruieren sucht, also seit ,Toward a General Theory of Action' (1951) (vgl. auch Habermas 1981a: 36; Luhmann 1980a: 8; Schluchter 1980: 111). Um die Verankerung der Medientheorie in den Grundlagen der Parsons'schen Theorie-Architektur wie die Notwendigkeit ihrer Einführung zu verdeutlichen, sollen Parsons' basale Axiome kurz referiert werden.

In der systemtheoretischen Konstruktion wird die Handlung (unit act) als Subspecies innerhalb der Species lebender Systeme aufgefaßt. Korrelat des Konzepts lebender Systeme ist der Funktionsbegriff: Systeme müssen aus der Notwendigkeit der Bestandserhaltung ${ }^{2}$ heraus bestimmte Funktionen erfüllen.

Diese Funktionen lassen sich mittels einer einfachen Überlegung konkretisieren:

Parsons selbst hat die Bezeichnung, Strukturfunktionalismus' für seine Theorie mit der Begründung zurückgewiesen, daß Funktion und Struktur nicht derselben Abstraktionsebene angehören (vgl. Parsons 1970: 35). Als ,Schulbezeichnung، mag der Terminus jedoch angehen.

Zu den Unklarheiten der Bestanderhaltungsformel bei Parsons s. a. Luhmann (1968: 147f.).
Systeme existieren in Raum und Zeit. Die Abgrenzung von der Umwelt muß durch Austauschprozesse zwischen Innen und Außen aufrechterhalten werden; die Systemgrenze trennt dann höhere Komplexität und niedrigere Stabilität in der Umwelt von geringerer Komplexität und höherer Stabilität (oder Ordnung) im System. Diese Systemeigenschaft läßt sich auf der Raumachse als externalinternale Differenz abbilden.

Die Differenz von Innen und Außen muß dauerhaft aufrechterhalten werden; die Prozesse, die die Aufrechterhaltung gewährleisten, beanspruchen Zeit. Die zukünftige Bestandserhaltung des Systems muß also ebenfalls sichergestellt werden. Die Zeitachse wird also durch eine instrumentellkonsumptorische Differenz konstituiert.

Durch Kreuztabellierung der beiden dichotomen Achsen läßt sich auf logisch-deduktivem Wege eine vierfache Klassifikation und Spezifizierung der Bestandserhaltungsfunktion ableiten.

Die Innenfelder der Kreuztabelle ergeben Parsons' AGIL-Schema systemischer Differenzierung, das Adaption (A) und Goal-Attainment (G), die auf das Verhältnis zur Umwelt bezogen sind, Integration (I) und Latent-Pattern-Maintenance (L), die auf system-interne Probleme bezogen sind, als die vier Grundfunktionen ausweist (vgl. Parsons 1956: 19, Fig. 1; 1970: 32; vgl. a. Gould 1976: 475). Für das AGIL-Schema erhebt Parsons den Anspruch universaler, quasi-axiomatischer Geltung:

„(..) the four-function scheme ist grounded in the essential nature of living systems at all levels of organization and evolutionary development, from the unicellular organism to the highest human civilization." (Parsons 1970: 35)

Die Technik der Kreuztabellierung hat weitreichende Auswirkungen auf die weitere Ausarbeitung der Theorie: Einmal legen Wahl und Plazierung der Randvariablen eine bestimmte Konkretisierung der Innenfelder von vornherein nahe (vgl. Luhmann 1980a: 11). Lidz (1981: 40ff.) warnt denn auch vor der simplen Umstellung, wie sie etwa Gould (1976) auf der Zeitachse vornimmt, wobei er als Argument die gewaltigen Folgen dieses Eingriffs ins Feld führt.

Zum anderen werden die Möglichkeiten funktionaler Differenzierung schon auf dieser Ebene festgeschrieben:

„Aufgrund der theoretischen Ableitung kann (und muß!) die Theorie postulieren, daß es nur diese Funktionen gibt. Das heißt: Jedes Handlungssystem muß sie (...) realisieren. Und das heißt: Jede Systemdifferenzierung erfordert eine Wiederholung dieses Schemas innerhalb 
der nach diesem Schema gebildeten Funktionssysteme." (Luhmann 1980a: 10)

Die Anwendung des Vierfunktionsschemas auf die Handlung ergibt folgende funktionale Systemdifferenzierung:

Das Kultursystem übernimmt die Funktion der Strukturerhaltung (L), das Sozialsystem die der Integration (I), das Persönlichkeitssystem reguliert die Zielerreichung $(\mathrm{G})$, der Verhaltensorganismus besorgt die Anpassung (A) (vgl. Parsons 1975b: 50f. Tab. 1).

Die Subsysteme des allgemeinen Handlungssystems tendieren selbst wiederum dazu, die Systemdifferenzierung im Inneren $\mathrm{zu}$ wiederholen. So differenziert sich das Sozialsystem (die Gesellschaft) in kulturelles Treuhandsystem (L), kommunale Gemeinschaft (I) (societal community; empirisch: Stamm, Volk, Polis, Nation; vgl. Parsons 1968b: 461), Politik (G) und Wirtschaft (A).

Parsons' in der Kreuztabellierung begründeter ,teleologischer Funktionalismus' hat zur Folge, daß sozio-kulturelle Evolution sich ausschließlich über Systemdifferenzierung vollziehen muß. Nimmt sozialer Wandel andere Formen an, kann er per definitionem nicht als evolutionäre Veränderung verstanden werden (vgl. Baum 1976b: 539) - soziokulturelle Evolution und Systemdifferenzierung werden äquivok. ${ }^{3}$

\section{Interpenetration und Medien als Integrationsmechanismen}

Es ist das Differenzierungskonzept als der Grundboden der gesamten Theorie-Architektur, das die Entwicklung der komplementären Theorien der Interpenetration (vgl. Jensen 1978; Luhmann 1977 und 1978, sowie, von Jensens und Luhmanns Interpretation abweichend ${ }^{4}$, Münch 1980a: 34ff.) und

${ }^{3}$ Eine ähnliche definitorische Äquivokation ergibt sich für die Formulierung von Prozessen gesellschaftlicher Rationalisierung (vgl. Stichweh 1980: 68).

${ }^{4}$ Anders als Jensen und Luhmann interpretiert Münch die Märkte zwischen den verwandten Subsystemen als Interpenetrationszonen. Er behandelt die Theorie der generalisierten Austauschmedien also als Teilgebiet des Konzepts der Interpenetration (vgl. Münch 1980b: 26ff.). Für Jensens und Luhmanns Interpretation spricht eher Parsons (1968a: 437). der Tauschmedien als logische Konsequenz impliziert (vgl. Luhmann 1980a: 14). ${ }^{5}$

„Das funktionale Gegenstück einer auf Differenzierung begründeten Evolution ist das Problem der Integration; und dieses Problem muß unabdingbar gelöst sein, um den Prozeß der Evolution tatsächlich gelingen zu lassen. Die Medien bilden die entscheidende Entwicklung von Mechanismen zur integrativen Stabilisierung und stellen damit die Lösung des funktionalen Gegenproblems der Differenzierung dar." (Jensen 1980a: 28)

"The need for generalized media of interchange ist a function of the differentiatedness of social structures: in this sense they are all partly integrative mechanisms." (Parsons 1968b: 471; ähnlich Parsons 1970: 30: Baum 1976a: 450; Cartwright/Warner 1976: 639)

Aus evolutionärer Perspektive gesehen folgt also die Ausdifferenzierung der Medien auf eine vorgängige Systemdifferenzierung, nicht umgekehrt (vgl. Luhmann 1976: 507). Da die Medien funktional auf die Probleme der Systemdifferenzierung bezogen sind, gibt es für jedes ausdifferenzierte Subsystem genau ein Medium, also auf jeder Differenzierungsebene jeweils genau vier: vier Medien auf der Ebene des allgemeinen Handlungssystems, je vier Medien für Kultursystem, Sozialsystem, Persönlichkeit und Verhaltensorganismus. Das heißt: für die Handlung (unit act) und ihre vier funktionalen Subsysteme müssen theoretisch zwanzig Medien angenommen werden.

\section{Die Medientheorie zwischen Austausch und Interaktion}

Für die Struktur der Medien ist entscheidend, daß auf der Ebene des Sozialsystems, dem historischen Ausgangspunkt der Konzeption ${ }^{6}$, die Beziehungen zwischen den ausdifferenzierten Subsystemen doppelt bestimmt sind:

In einer Hinsicht ist das Sozialsystem um die Muster der Interaktion individueller Akteure herum organisiert. Das gilt auch für Kollektive und Organisationen, die nur über individuelle, autorisierte ,Sprecher' miteinander interagieren können (Parsons 1970: 36, Anm. 10). Diese Interaktion nimmt die Form von Kommunikation an. Medien sind

Zum Verhältnis von Interpenetration und Austauschmedien bei Parsons s. a. Luhmann (1978: 300).

'Baum zufolge (1976a: 449) licgt mit ein Grund für die Probleme der Medientheorie in dem Umstand, daß die Theoriearbeit nicht auf der allgemeineren Ebene des Handlungssystems begonnen wurde. 
hier Interaktionsmedien (in 1968a: 440 spricht auch Parsons schon von ,media of communication'), die Information, das heißt Selektionen, von einem Sender zu einem Empfänger übertragen.

Auf der anderen Seite liefern die Subsysteme die Komponenten, aus denen sich Funktion und Einheit des ihnen übergeordneten Ursprungssystems zusammensetzt (vgl. Parsons 1968b: 460). Da die Subsysteme funktional auf jeweils eine Funktionskomponente spezialisiert sind, sind sie für deren Produktion auf Ressourcen, also auf Faktoren und auf Produkte aus den drei verwandten Subsystemen angewiesen. (Dahinter verbirgt sich das klassische Konzept von Arbeitsteilung.) Zwischen den Subsystemen einer Differenzierungsebene laufen also Input- und Output-Ströme hin und her. Medien dienen hier der Vermittlung von Input und Output und werden dementsprechend auch als Austauschmedien konzipiert. ${ }^{7}$ (Vgl. Parsons 1970: 36ff.)

Sowohl die mit jeder Interaktion implizierte doppelte Kontingenz (Parsons 1968a: 463) als auch die Aufrechterhaltung der intersystemischen Austauschprozesse werden also für das System zum funktionalen Problem, das nur durch die Etablierung eines Mediums zu lösen ist. Ein und dasselbe Medium ist dabei für beide Probleme zuständig. Die Unterscheidbarkeit von Interaktionsproblem und Austauschproblem hängt allein von der Referenz ab; das Medium bleibt dasselbe, auch wenn der Blick einmal die Vermittlung der Interaktion und einmal die Vermittlung des Austauschs erfaßt. Mit dem Wechsel der Referenz verschwindet jedoch gleichzeitig das vormals fokale Problem, so daß es sich bei der Thematisierung von Interaktion oder Austausch um ein ausschließendes „oder“, um eine Kontravalenz handelt. Das könnte als Indiz dafür gewertet werden, daß Parsons die Integration von Handlungstheorie und Systemtheorie doch nicht vollständig bruchlos gelungen ist (vgl. Habermas 1981a). Die Doppelstruktur der systeminternen Prozesse: Interaktion und Austausch, und die daraus folgende Doppelstruktur der Medien sind mit die Ursache für etliche der Ambiguitäten, die das Konzept der symbolisch generalisierten Medien durchziehen.

\footnotetext{
In der ersten Studie Parsons, in der die Medien eine Rolle spielen, ist nur vom Austauschaspekt, nicht explizit von Interaktion die Rede (vgl. Parsons 195: $70 \mathrm{ff}$.$) .$
}

\section{Parsons' Nomenklatur}

In Parsons' Nomenklatur werden nun den Systemen der Handlung und ihren Subsystemen folgende Medien zugeordnet:

Das zentrale Medium des allgemeinen Handlungssystems ist die Sprache - besser: symbolic meaning oder einfach meaning (vgl. Parsons 1978: 394); auf der Subsystemebene des allgemeinen Handlungssystems werden der Kultur ,definition of situation", dem Sozialsystem ,affect', dem Persönlichkeitssystem ,performance capacity und dem Verhaltensorganismus, intelligence“ als Medien beigestellt.

Im ausdifferenzierten Sozialsystem sind dann dem kulturellen Treuhandsystem Wertbindungen, dem Gemeinschaftssystem Einfluß, der Politik Macht und der Wirtschaft Geld als Medien zugeordnet (vgl. auch das vollständige Medienschema bei Münch 1980b: 20, Fig. 5; u. 22, Fig. 6; ähnlich bei Jensen 1984: 163; für alternative Medienvorschläge auf der Ebene des allgemeinen Handlungssystems s. a. Lidz 1981: 77 f., nämlich Kollektive Repräsentationen (L), Definition der Situation (I), Objektrepräsentationen $(\mathrm{G})$ und Intelligenz nach Piaget statt nach Skinner (A)).

\section{Sprache und Geld in der Medientheorie}

Die erwähnte Doppelstruktur der Medien, die sich dem Umstand verdankt, daß die Medien funktional auf Kommunikation und auf Austausch bezogen sind, spiegelt sich in der Spezifizierung ihrer Charakteristika wider: ihre Eigenschaften und Rahmenbedingungen lassen sich teilweise jeweils einem der beiden funktionalen Bezugsprobleme zuordnen; zentrale Punkte sind so konzipiert, daß sie durch Uminterpretation scheinbar beiden Aspekten gerecht zu werden vermögen.

Die Charakteristika der Medien gewinnt Parsons durch eine Abstraktion, die von jeweils einem für paradigmatisch genommenen Grundmuster der Medien ausgeht. Dem Austauschaspekt entspricht als Grundmuster das Geldmedium, der Kommunikations- oder Interaktionsaspekt wird auf der Grundlage eines linguistischen Modells, also auf Sprachbasis erfaßt. Dem Anspruch nach handelt es sich teils um ein Verhältnis der Ergänzung, teils wird die Sprache als das grundlegendere Modell ausgewiesen:

„Money (. . .) can be considered as a special case of a very general phenomenon: language. It is in fact a very highly spezialized language." (Parsons 1968b: 470) 
Es läßt sich jedoch zeigen, daß die Grundmodelle des Medienkonzepts miteinander inkompatibel sind, also in der Theorie konkurrieren und so zu Unstimmigkeiten führen - diese Unstimmigkeiten brechen nur deshalb nicht offen durch, weil für die angewandten Analysen letzten Endes doch das Geldmodell den gemeinsamen Nenner von Kommunikations- und Austauschprozeß bildet (Vgl. Coleman 1963: 65).

\section{Eigenschaften der Medien}

Die Medieneigenschaften lassen sich zum Teil dem linguistischen Schema von Code und Botschaft (message) zuordnen. Auf der Message-Ebene sind die Medien durch Symbolgebrauch gekennzeichnet. Symbole sind prinzipiell intrinsisch wertlos, sie sind, nothing of value'. Mit der Symbolisierung geht eine Generalisierung einher: Die Bedeutung und Verwendung der Symbole wird vom partikularen Kontex unabhängig, sie sind in einer Vielzahl von Situationen verwendbar. Weiter müssen die Symbole zirkulieren können. Zirkulierbarkeit der Symbole bedeutet - und hier findet schon eine monetäre Uminterpretation linguistisch-semantischer Symbole statt -, daß man Mediensymbole besitzen, ausgeben, horten, sparen und investieren kann (vgl. Loubser 1981: 364). Obwohl selbst intrinsisch wertlos, sollen die Symbole doch im Austausch und in der Kommunikation motivierend wirken können. Motivationswirksamkeit kommt ihnen zu, insofern sie auch der Wertaufbewahrung dienen; sie sind ,store of value", stehen also als Tauschwerte für Gebrauchswerte. Ihr Tauschwertcharakter verlangt zum einen die Existenz einer Sicherheitsgrundlage, durch die die zirkulierenden Symbole gedeckt sind; zum anderen muß es möglich sein, die intrinsisch wertlosen Tauschwerte real gegen Gebrauchswerte, gegen intrinsic satisfiers ${ }^{8}$ einzutauschen. Die Mediensymbole müssen also in diesem Sinne konvertierbar sein. Da erst die als Austauschprozesse aufgefaßten Relationen zwischen den funktional ausdifferenzierten Subsystemen die Identität des Ursprungssystems konstituiert, muß ferner jedes Medium in alle anderen Medien auf gleicher Differenzierungsebene konvertierbar sein (vgl. Parsons 1970: 42; Baum 1976c:

${ }^{8}$ Bei den intrinsic satisfiers handelt es sich um den ,Referent' des Symbols; der Bereich möglicher Referenten erstreckt sich von Objekten über Objekteigenschaften bis zu Beziehungen zwischen Aktoren (vgl. Turner 1968: 122).
581). Der Aufgabe der Wertaufbewahrung auf der Symbolebene entspricht ein komplementärer Zug auf der Code-Ebene: Medien sind nicht nur ,store of value', sondern auch ,measure of value', dienen also notwendig auch als Wertmaßstab. Wertmaßstab sind sie in zweierlei Hinsicht:

Zum einen verknüpfen sie das leitende Wertprinzip ihres Subsystems, in dem dessen spezialisierte Funktion verankert ist, und das ebenfalls zur Codestruktur gehört, mit den Bestandteilen, die als Produktionsfaktoren in die Verwirklichung des Wertes eingehen. Anders gesagt, dient der Wertmaßstab der Relationierung von Tauschwerten oder ,values in exchange' mit den Gebrauchswerten oder ,values in use'; und zwar derart, daß Medien als Mechanismen rationaler Wahl wirken können (vgl. Baum 1976a: 461): sie ermöglichen eine subsystemspezifische Rationalität (vgl. Stichweh 1980: 75). Zum anderen ermöglicht der Wertmaßstab die Skalierung der Symbole, also der, values in exchange'. Auf dieser Ebene treten die Mediensymbole in der Form wählbarer Quantitäten auf (vgl. Jensen 1980: 17 und 19).

Neben dem Wertmaßstab, dem Wertprinzip und der subsystemspezifischen Rationalität gehören zur Code-Struktur noch ein Koordinationsstandard und der eigentliche operative Code. Der operative Code besteht aus der normativen Hintergrundstruktur des Mediums, durch die die legitimen Formen des Mediengebrauchs festgesetzt und illegitime Formen ausgeschlossen und sanktioniert werden. Konkret wird hier darüber entschieden, welche Klassen von Objekten durch die Mediensymbole erfaßt werden und in welchen Klassen von Situationen das Medium eingesetzt werden darf (vgl. Baum 1976a; Jensen 1980: 15; Jensen/Naumann 1980: 81). Die Verknüpfung der Medien mit der normativen Hintergrundstruktur wird dabei auf der Institutionsebene vollzogen:

„Institutionen (. . .) sind Komplexe von normativen Regeln und Prinzipien, die entweder kraft Gesetz oder durch andere Mechanismen sozialer Kontrolle zur Steuerung sozialen Handelns und sozialer Beziehungen dienen. (. . .) jedes Medium ist mit einem funktional definierten Institutionskomplex verknüpft." (Parsons 1980d: 232)

Medien sind ferner dadurch charakterisiert, daß sie nicht Nullsummenbedingungen unterworfen sind: Die umlaufende Symbolmenge ist nicht endgültig fixiert, es existieren vielmehr für jedes Medium Mechanismen, die der Kreditschöpfung ana$\log$ funktionieren, also eine Nettozunahme des zirkulierenden Quantums von Symbolen bewir- 
ken. Für den Vollzug der ,Symbolschöpfung' sind in jedem System bankanaloge Einrichtungen vorhanden (Parsons 1980a: 98f.; 1980d: 236f.).

\section{Funktionen der Medien}

Die Vielzahl von Eigenschaften und Strukturen, die den Medien zugeschrieben werden, ist daraufhin konzipiert, daß Medien (1) individuelle Kommunikation vermitteln und sanktionieren können, (2) den Ressourcen- (Faktoren-) und Produktaustausch zwischen ausdifferenzierten Subsystemen tragen und (3) überdies Grenzerhaltung und Identität der Systeme im Verhältnis zu ihrer Umwelt garantieren, indem sie Komplexität regulieren, sie also einerseits reduzieren, aber auch im Bedarfsfall produzieren (vgl. Baum 1976b: 535ff.).

Unter dem Kommunikationsaspekt kann man Medien als Mittel definieren, mit denen man Intentionen (also eine spezielle Art selektiver Information) übermittelt und gleichzeitig beim Interaktionspartner das Motiv erzeugt, die erwünschte komplementäre Handlung zu vollziehen. Der motivationswirksame Grund, die Selektion zu übernehmen, ist das Medium selbst - ,the medium is the message“ (vgl. Jensen 1980: 15). „Medien haben den imperativen Modus gemeinsam, das heißt, sie führen zu Resultaten, statt bloß Information zu übertragen. Sie konfrontieren das Objekt (alter) mit einer Entscheidung, die eine Reaktion erfordert - etwa Annahme oder Ablehnung eines Geldangebots." (Parsons 1980b: 144) - Und sanktionieren Alters Reaktion. Oder, wie Cartwright/Warner sagen: „They are ways of getting things done.“ (1976: 640)

Unter dem Austauschaspekt gesehen, wirken Medien als Tauschwerte, die auf Märkten zwischen den Subsystemen gegen Inputs, das heißt gegen Produkte und Ressourcen ausgetauscht werden. Auch die Austauschbeziehungen zwischen den Subsystemen wie die Marktstrukturen sind durch das Vierfunktionsschema festgelegt: Die vier Medien jeder Systemebene regulieren einen Austauschproze $\beta$, der sich auf sechs Märkten abspielt.

Hierfür ist ihre Konvertierbarkeit vorausgesetzt, denn Inputs wie Outputs werden in Medienform angeboten. Ressourcen (Faktoren) werden auf dem Markt in Form des Mediums ihres Zielsystems angeboten; Produkte kommen in Form des Mediums ihres Zielsystems auf den Markt (vgl. Parsons 1980a: 119). Insofern konvergiert die Konvertierbarkeit der Medien untereinander mit ihrer Konvertierbarkeit in intrinsic satisfiers.

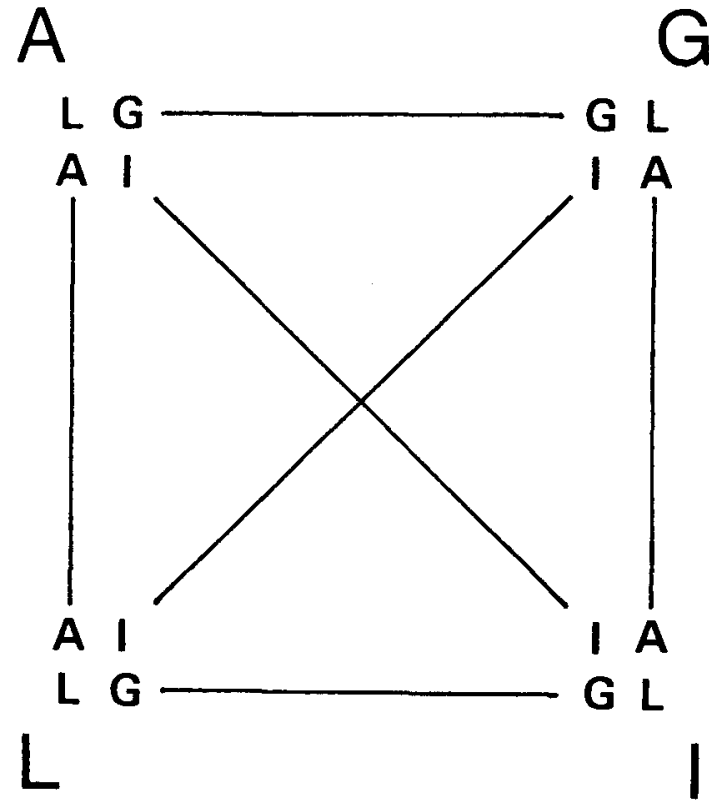

Abbildung 1 intersystemische Märkte und Austauschbeziehungen.

„From the perspective of any one functional subsystem, (. . .) the inputs of shares of product received from the neighboring subsystems always come in the form of that medium functionally specialized for internal processes of the recipient subsystem. The obverse holds for factor inputs. They always come in the form of the media functionally specialized for internal processes of the respective emitting subsystems." (Baum 1976a: 459)

Da zwischen den Subsystemen stets sowohl Produkte als auch Faktoren (Ressourcen) gegeneinander ausgetauscht werden, findet zwischen zwei benachbarten Subsystemen stets ein doppelter Austausch statt.

„Hence inputs come in forms of media favoring the functionspecific medium of the recipient subsystem in a ratio of 3 to 1. " (Baum 1976a: 459)

Ein wesentlicher Vorteil symbolisch generalisierter Medien sowohl in Hinblick auf Kommunikation wie in Hinblick auf Austausch ist, daß ihre Verwendung Information und damit auch Zeit spart, die in komplexen Systemen ebenfalls zu einer knappen Ressource wird (vgl. Baum 1976c: 580). In der Kommunikation wird Zeit gespart, insofern Alter die Selektion übernimmt, ohne den Selektionsproze $B \mathrm{zu}$ wiederholen. Beim mediengesteuerten Austausch auf Märkten wird Zeit gespart, insofern die Medien als reine Tauschwerte die Überwindung des eigentlichen Tauschhandels 
(barter) ermöglichen. Hierfür sind Symbolisierung, also intrinsische Wertlosigkeit, Generalisierung, also Kontextunabhängigkeit, und implizit auch die Quantifizierbarkeit der Symbole vorausgesetzt. Tauschhandel (barter) als Gegenstück des medienvermittelten Austausches bedeutet den direkten Austausch von intrinsisch wertvollen Outputs gegen ebensolche Inputs. Bedingung dafür ist ein Zusammenfallen der Bedürfnisse beziehungsweise des Systembedarfs. Tauschhandel mag in primitiven Gesellschaften angehen, da diese als segmentär differenzierte aus strukturell identischen Einheiten mit identischen Bedarfsstrukturen bestehen (vgl. Luhmann 1980b: 17).

Funktional differenzierte Systeme mit einem hohen Grad an intersystemischer Arbeitsteilung sind jedoch darauf angewiesen, daß die durch Spezialisierung der Subsysteme auf bestimmte Funktionen gewonnenen Vorteile nicht durch zeitraubende und riskante Austauschprozesse wieder verloren gehen (Parsons 1970: 39). Für sie ist Tauschhandel eine Regression und damit bestandsgefährdend.

Die Austauschmedien können in dieser Hinsicht auch als Mechanismen verstanden werden, die gerade weil und indem sie durch das ganze System zirkulieren - für ihr Bezugssubsystem auch die Funktion übernehmen, die Komplexität der Umwelt, das heißt ihrer Nachbarsysteme, zu reduzieren (vgl. Baum 1976a: 455).

Komplexität reduzieren die Medien ebenfalls für individuelle Akteure: Das ,Ausgeben“ von $\mathrm{Me}$ diensymbolen bedeutet für den Besitzer des Mediums ein Festlegen seines Handelns, also eine Selektion, die eine Bindung seiner Kapazitäten und den Ausschluß anderer Möglichkeiten impliziert. Baum zufolge wird in diesem Vorgang die Komplexität jedoch nicht vernichtet, sondern erhalten: Egos Bindung seiner Kapazität an eine bestimmte Selektion bedeutet für den Interaktionspartner Alter eine Erhöhung seiner Kapazität, eine positive Erweiterung der Kontingenz und für das System insgesamt eine Steigerung der internen Komplexität. Ersichtlich dient hier das Geldmedium als Muster: Egos Ausgabe schränkt seine künftigen Möglichkeiten ein und legt ihn auf einen Kaufakt fest; der Empfänger Alter wird dagegen in die Lage versetzt, in Zukunft unter mehr und erweiterten Möglichkeiten zu wählen, da er nun über ein größeres Quantum des Mediums verfügen kann. Baum sieht darin einen Mechanismus, der erwünschte, systeminterne Komplexität produziert und der bei sämtlichen Medien nachgewiesen werden kann (vgl. Baum 1976b: 538, 554). Anders
Luhmann: er sieht in der Komplexitätserhaltung durch das Geldmedium einen einzigartigen Mechanismus. Der Transfer aller anderen Medien bindet im Gegensatz zum Geldmedium beide Seiten, führt also nur zur Reduktion von Komplexität. Entsprechend besteht in den Systemen mit nichtmonetären Medien ein komplementärer Bedarf an gegenläufigen Mechanismen der Komplexitätsproduktion (vgl. Luhmann 1976: 51).

\section{Das ungeklärte Verhältnis von Interaktion und Austausch als Effekt der Konkurrenz von Mikrosoziologie und Makrosoziologie}

Als eine der Ursachen für die Ambiguität der Medientheorie wurde schon das Faktum ausgemacht, daß auf der Ebene des Sozialsystems alle Prozesse doppelt determiniert sind, nämlich sowohl als Interaktion (oder Kommunikation), als auch als Austausch aufgefaßt werden können. Diese zweifache Interpretationsmöglichkeit sämtlicher Prozesse könnte ein Indiz dafür sein, daß Parsons das Verhältnis von Systemtheorie und Handlungstheorie in seiner Theoriearchitektur doch nicht endgültig geklärt hat und daß die Ambiguität der Medientheorie nur die Konkurrenz zwischen Handlung und System auf der Grundlagenebene widerspiegelt. (Eine Konkurrenz von Handlungstheorie und Systemtheorie in Parsons' Werk ist ebensooft bestritten wie behauptet worden; exegetisch dürfte das Problem nicht mehr zu lösen sein.) Mindestens lassen sich die beiden Aspekte der Systemprozesse den Ebenen (und der Konkurrenz) von Mikrosoziologie und Makrosoziologie zuordnen, wie Luhmann es getan hat:

Die mikrosoziologische Theorie der Interaktion ist dabei auf den Begriff der doppelten Kontingenz aufgebaut, dessen Einführung für Parsons die logische Konsequenz des Interaktionskonzeptes ist (vgl. Parsons 1968a: 436) (hier setzt auch Luhmanns Fassung der Medientheorie an). Die makrosoziologische Theorie der Systemdifferenzierung ist dagegen auf den Begriff des Austauschs bezogen. Luhmann zufolge beherrscht schließlich die makrosoziologische Theorie das Feld; ,doppelte Kontingenz' wird von ,double exchange" verdrängt (vgl. Luhmann 1976: 508). Innerhalb der Medientheorie spiegelt sich das Verhältnis von Mikrosoziologie und Makrosoziologie in der erwähnten Konkurrenz der beiden paradigmatischen Modelle Sprache und Geld. Auf der Ebene des Austauschs zwischen Subsystemen werden die Me- 
dien geldanalog konzipiert, auf der Ebene der Interaktion werden sie als Spezialsprachen aufgefaßt, also sprachorientiert konzipiert.

„Auf der Ebene individueller Akteure fungieren Medien als Spezialsprachen, die eine bestimmte, spezialisierte Art von Kommunikation oder Transaktion durch spezifische symbolische Generalisierung modellieren und steuern. (. . .) Dieses sprachanaloge Verständnis wird aber überlagert und zum Teil ersetzt durch ein geldanaloges Verständnis. Systemreferenz ist nicht die Ebene individueller Akteure, sondern die Ebene ausdifferenzierter Teilsysteme. Hier besteht die Funktion der Medien darin, Austauschprozesse zwischen den Teilsystemen zu ermöglichen und zu steuern. Nach dieser Funktionsbestimmung kreisen die Medien nicht so sehr innerhalb der spezialisierten Teilsysteme, sondern sie zirkulieren zwischen den Teilsystemen als Mechanismus der Integration des Ganzen." (Willke 1982: 123f.)

\section{Die grundlagentheoretische Einordnung des Geldmediums in das Sprachmodell}

Parsons selbst hat das Geldmedium seit ,On the Concept of Influence' (1980b) in das Sprachmodell eingeordnet, indem er es als Spezialsprache auswies (siehe oben). Sprachanalog ist das Geldmedium für Parsons aus folgendem Grund:

„It operates at the symbolic level and its primary function is communication, though of a special, normative sort. The ,monetary' system ist a code, in the grammaticalsyntactical sense. The circulation of money is the ,sending off" messages. " (Parsons 1968b: 470)

Diese Einordnung bleibt nicht konzeptuelle Sackgasse, wie Coleman (vgl. 1963: 65) meint. Für die Integration des Geldmodells in die an Sprache orientierte Konzeption der Medien - und damit auch für die Vermittlung von Interaktionsebene und Austauschebene - spielt der Begriff der Interpenetration eine zentrale Rolle (vgl. Willke 1982: 124). Die Anwendung des Interpenetrationsbegriffs selbst ist jedoch nur aufgrund Parsons' spezifischem Verständnis der Sprache möglich; Parsons sieht in der Sprache im Grunde keinen Modus der Interaktion, er ordnet sie vielmehr dem Kuitursystem zu:

„The most important single condition of the integration of an interaction system is a shared basis of normative order. (. . .) The concept of a shared basis of normative order is basically the same as that of a common culture or a ,symbolic' system. The prototype of such an order is language. A language involves a code, consisting of generalized norms which define, correct' speech or writing, as the basis for using symbols to formulate and transmit messages. (. . .) Language (. . ) is not a primary normative constituent of social systems (. . .) but is a

\begin{tabular}{|c|c|c|c|}
\hline & & $\begin{array}{l}\text { Kultur } \\
\text { Institutio- } \\
\text { nalisierung } \\
\text { Situations- } \\
\text { kanal }\end{array}$ & $\begin{array}{l}\text { Interna- } \\
\text { lisierung } \\
\text { Intentions- } \\
\text { kanal }\end{array}$ \\
\hline \multirow{2}{*}{ positiv } & Modus & Anreiz & Überredung \\
\hline & Medium & Geld & EinfluB \\
\hline \multirow[t]{2}{*}{ negativ } & Modus & $\begin{array}{l}\text { Eischüch- } \\
\text { terung }\end{array}$ & $\begin{array}{l}\text { Appel an } \\
\text { Wertbindungen }\end{array}$ \\
\hline & Medium & Macht & Wertbindungen \\
\hline
\end{tabular}

Abbildung 2 Das Sanktionsschema der Medien des Sozialsystems (vgl. u.a. Parsons 1980b: 147).

primary normative constituent of distinct cultural systems. "(Parsons 1968a: 437; Hervorhebung von mir, J. K. .) ${ }^{9}$ Oder ausdrücklich: „(. . .) language should be treated as a part of culture." (Parsons 1982: 56).

Durch diese Zuordnung wird der Sprache der direkte Bezug auf die Prozeßebene von Handlung, und damit auch von Interaktion, genommen; sie gehört nur noch zu deren strukturellen Rahmenbedingungen oder Steuerungsfaktoren.

Durch Interpenetration werden bei Parsons die Strukturen von Kultursystem, Sozialsystem und Persönlichkeitssystem miteinander vermittelt. Interpenetration bedeutet hier, vereinfacht gesagt, die Institutionalisierung kultureller Muster im Sozialsystem und ihre Internalisierung im Persönlichkeitssystem (Parsons 1968a: 437), der Interpenetrationsbegriff ist zunächst einfach Gattungsbegriff zu den Begriffen Institutionalisierung und Internalisierung. Da Medien als Spezialsprachen mit imperativem Modus aufgefaßt werden, kann man sie zunächst danach klassifizieren, ob ihre Sanktionen negativ oder positiv sind. Auf der anderen Seite ist die mit Sprache identifizierte normative Ordnung

9 Zur Kritik an Parsons' Differenzierung zwischen Kultursystem und Sozialsystem s. u. a. Lipp (1979: 465ff.) und Luhmann (1980: 16): „Das Kultursystem ist (nach Parsons' Definition: ,Culture consists in codified systems of meaningfull symbols; and those aspects of action directly oriented to problems of meaningfullness of such symbols.' J. K.) zwar ein Teilsystem des Handlungssystems, besteht aber selbst nicht aus Handlungen, sondern aus Symbolen. Würde man konsequenter formulieren: Kultursystem sei das Handlungssystem, soweit es sich auf Symbole beziehe, läge auf der Hand, daß dies kein analytisch, geschweige denn real ausdifferenzierbares Teil sein kann, da Handlung nur durch Symbolgebrauch Handlung sein kann." 
sowohl in der Persönlichkeit (als Teilsystem des Individuums) internalisiert, als auch ein dem Individuum äußerliches soziales Faktum im Sinne Durkheims, eben insoweit, als sie im Sozialsystem institutionalisiert ist. Institutionalisierung normativer Muster macht Sanktionen möglich, die Alters Situation verändern; ihre Internalisierung macht Sanktionen möglich, die auf Alters Intentionen einwirken. Durch Kreuztabellierung von Kulturaspekt und Sanktionsaspekt gewinnt Parsons ein Schema zur Klassifikation der Medien, das zugleich allen Innenfeldern einen unausgesprochenen Bezug auf die Sprache verleiht, eben insofern die Randvariable Kultur mit Sprache identifiziert werden kann. ${ }^{10}$

Solchermaßen kann Geld als Spezialfall in Parsons' kulturalistisches Sprachkonzept eingeordnet werden - jedoch erfaßt das Klassifikationsmodell nur die Ebene der Interaktion, also das Problem der Handlungskoordination zwischen Ego und Alter. Für die Austauschebene wird der Nachweis, daß Geld als Spezialsprache verstanden werden kann, nicht erbracht.

\section{Die monetäre Umdeutung des Sprachmodells}

Trotz gegenteiliger Äußerungen werden auf der Ebene des Austauschs zwischen funktional differenzierten Subsystemen sämtliche Medien als monetäre Mechanismen konstruiert; zuguterletzt wird auch die sprachliche Kommunikation als Input/ Output-Austausch begriffen, die Sprache wird also durch eine informationstheoretische Wendung unter das Geldmodell subsummiert. Wenn man die Charakteristika der Medien genauer auf ihre Stellung zwischen Geldähnlichkeit und Sprachähnlichkeit hin untersucht, ist das Überwiegen und die funktionale Dominanz derjenigen Aspekte, die Verallgemeinerungen des Geldmechanismus darstellen ebensowenig zu übersehen, wie die monetäre Umdeutung genuin sprachlicher Komponenten.

Zunächst fällt auf, daß unter den abstrakten Eigenschaften, die allen Medien zugeschrieben werden, Topoi auftauchen, die in den Wirtschaftswissenschaften als die Grundfunktionen des Geldes

${ }^{10}$ Die Klassifikation der Medien durch diese Form der Kreuztabellierung findet sich schon in ,On the Concept of Power' (1980a: 73); man beachte jedoch die an Durkheim orientierte Uminterpretation der situationalen und intentionalen Randvariable bei Parsons 1968a: 437; vgl. auch 1980c: 193ff.). behandelt werden: Tauschmittelfunktion, Rechenfunktion (measure of value) und die Funktion der Wertaufbewahrung (store of value) (vgl. u. a. Ehrlicher 1975: 353).

Weiter ist auch das Marktmodell eingestandenermaßen eine Verallgemeinerung des wirtschaftswissenschaftlichen Modells:

„Generalized from the economic case, a market could be defined as a social system specialized for mediating interests in which there are institutionalized expectations of willingness to exchange disposable resources for a medium and vice versa under a set of rules for setting terms and for the rights and obligations assumed and relinquished in the process. "(Baum 1976a: 457)

Auch der Mechanismus der Kreditschöpfung, also der Giralgeldschöpfung durch Banken, soll für alle Medien verallgemeinerbar sein; für die nicht-monetären Medien müssen deshalb ebenfalls bankanaloge Einrichtungen zur Verfügung stehen, die die umlaufende Symbolmenge vermehren und dadurch Nullsummenbedingungen überwinden können.

Und schließlich sollen alle Medien der Möglichkeit inflatorischer und deflatorischer Veränderungen unterworfen sein ${ }^{11}$. Die Anwendbarkeit und Verallgemeinerbarkeit wirtschaftlicher Phänomene und Konzepte wie Symbolschöpfung und Inflation beziehungsweise Deflation ist jedoch daran gebunden, daß die Mediensymbole bestimmte Eigenschaften haben. Eben diese Eigenschaften aber machen es gleichzeitig unmöglich, Mediensymbole als sprachliche Symbole (der Terminus, sprachliches Symbol' ist an sich unbefriedigend; es handelt sich genauer genommen nur um bestimmte Klassen von Ausdrücken, die sich aber nicht mit dem Symbolbegriff decken) oder, allgemeiner gesagt, Medien als Spezialsprachen zu interpretieren:

Die Mediensymbole müssen prinzipiell knapp, ihre umlaufende Menge muß begrenzt sein. Insofern

\footnotetext{
1] Merkwürdigerweise gibt es in Parsons' Medientheorie keine ausgearbeitete Preistheorie, sondern nur die Annahme von Gleichgewichtszuständen zwischen Inputs und Outputs (vgl. Baum 1976a: 452; auch Habermas hat das Fehlen eines Äquivalents für Preise registriert, vgl. 1981b: 86). Eine Folge dieses Defizits ist, daß die Bestimmung der Medien als Mechanismen rationaler Wahl ein Postulat bleibt. Konkretisierbar wäre der postulierte Rationalitätsgewinn erst, wenn nicht nur im Wirtschaftssystem, sondern auch in den anderen Subsystemen des Sozialsystems Preise die Funktion der Lenkung des Ressourceneinsatzes übernehmen würden.
} 
herrschen auch in der Wirtschaft hinsichtlich des Geldmediums notwendig Nullsummenbedingungen; und Kreditschöpfung wird überhaupt erst dadurch möglich, daß Knappheit quantifiziert und als Prinzip der Summenkonstanz operationalisiert werden kann (vgl. Luhmann 1984: 319; Loubser 1981: 369).

An Inflation und Deflation der Medien läßt sich die Notwendigkeit der Annahme einer knappen Symbolmenge eindrücklich demonstrieren. Inflation und Deflation sind, grob gesagt, Veränderungen in den Proportionen zwischen Geldmenge und der Menge an Gütern und Dienstleistungen (vgl. a. Jensen/Naumann 1980: 96). Schwankungen drükken sich in der Veränderung des Preisgefüges aus: Deflation bedeutet einen allgemeinen Preisrückgang, dem zunächst ein Steigen des Geldwertes korrespondiert; umgekehrt besteht Inflation in einem allgemeinen Preisanstieg, dem ein Wertverlust des Geldes entspricht.

Nur weil die Geldmenge knapp ist und sein muß, können Inflation und Deflation zu volkswirtschaftlichen Katastrophen wie Hyperinflation und Depression führen. Erst dann kommt es zur Flucht in Sachwerte (intrinsic satisfiers) und zur Rückkehr zum Tauschhandel (vgl. Cartwright/Warner 1976: 644f.). Dementsprechend müssen auch die Symbolmengen der nichtmonetären Medien knapp sein, wenn es sinnvoll sein soll, auch dort von Inflation und Deflation des Mediums zu sprechen.

Wenn jedoch die Medien als Spezialsprachen konzipiert werden, ist die Symbolmenge nicht begrenzbar: Jeder kompetente Sprecher kann beliebige Mengen von Symbolen erzeugen; die Vorstellung, ein Individuum könne eine begrenzte Menge semantischer Symbole ,besitzen', ist offensichtlich widersinnig. Genau das aber muß eine sprachorientierte Medientheorie behaupten, wenn sie zusätzlich mit wirtschaftswissenschaftlichen Begriffen wie Inflation und Deflation arbeiten will. So zum Beispiel Baum:

„Inflation connotes a change in the amount of symbols expended for a given amount of unit control over, real things' in the direction of increasing the symbols necessary to exercise this control. The change can be thought of as taking place between two points in time. Whereas at point $t_{1}$ one needed only $x$ amount of symbols, at $t_{2}$ one needs $x+n$ symbols to control the same amount of intrinsic satisfiers. In the case of influence whereas at $t_{1}$ it took but 10 sentences with $\mathrm{x}$ words to successfully suggest a strategy of therapy to a patient, at $t_{2}$, it takes 20 sentences with $\mathrm{x}+\mathrm{n}$ words to get compliance. Deflation is simply the reverse: Fewer symbols control what took more symbols before." (Baum 1976a: 461)

Wenn man eine Begrenzung von semantischen Symbolen (Baums Konzeption folgend, wohl besser: von Sprechakten), also Knappheit der Symbolmenge, annehmen will, dann allenfalls, indem man die ,Erzeugung' solcher Symbole an Regeln knüpft, die den Sprecher einschränken. Solche Regeln können nicht konstitutiv (also handlungsstiftend) sein - es muß sich vielmehr um regulative Regeln (also um nicht-konstitutive, statutorische Restriktionen im Sinne Shwayder's (vgl. 1965: 261)) handeln. Regulative Regeln aber sind, wenn sie Verbindlichkeit sollen beanspruchen können, notwendig an Mitgliedschaftsbedingungen von Organisationen gebunden (vgl. Grünberger 1981: 110f.). Im Falle des Machtmediums könnte man dann sagen, daß Amtsinhaber nicht beliebig Befehle ,erzeugen' können, sondern nur solange sie sich dabei regelkonform verhalten ${ }^{12}$. Das aber hie$\mathrm{Be}, \mathrm{da} ß$ die Symbolmenge, und damit auch inflationäre und deflationäre Prozesse, mit den Codestrukturen des Mediums verknüpft würden - bei Macht also mit Amtsinhaberschaft und den Mitgliedschaftsbedingungen als Regeln legitimen Medieneinsatzes ${ }^{13}$.

Wirtschaftliche Inflations- und Deflationsprozesse spielen sich jedoch, wenn man sie medientheoretisch analysiert, nur auf der Message-Ebene statt. Da sie nur das Verhältnis der Symbolmenge zur Menge der intrinsic satisfiers betreffen, bleibt die Code-Ebene davon unberührt - genauso wie die Code-Ebene auch vom ,Ausgeben' der Symbole unberührt bleibt. Die Symbolmenge darf schon deshalb nicht von einem an Mitgliedschaft gebundenen Regelbegriff abhängig sein, da es sonst um die Zirkulierbarkeit des Mediums durch das gesamte Sozialsystem geschehen wäre.

Die Dominanz des Geldmodells in der Medientheorie scheint insgesamt kaum bestreitbar zu sein. Wenn sie unauffällig bleibt und in ihrem Widerspruch zu Parsons' programmatischen Aussagen nicht offen hervortritt, dann deshalb, weil Parsons' Verständnis von Kommunikation und Sprache selbst einer monetären Uminterpretation entge-

${ }^{12}$ Es ist die Frage, ob dann Inflation und Deflation nicht einfach nur Devianz bei der Symbolerzeugung wären.

${ }^{13}$ Durch die Einführung von authority (jurisdiction of office) in den operativen Code (vgl. Parsons 1980a: 83; Baum 1976a: 467) ist diese Option prinzipiell möglich, wenn nicht gar nahegelegt. 
genkommt. Schon sein Modell der Kommunikation ist eher informationstheoretisch angelegt (vgl.

Parsons 1970: 36), trennt Kommunikation und Sprache, und muß deshalb die Funktion von Sprache systematisch unterschätzen:

„By portraying language as a technical invention which increases communication efficiency, Parsons implies that it is logically possible to conceive of social action and communication in the absence of language ( a claim which underestimates the close conceptual ties between communicating intelligibly and using a language correctly." (Cartwright/Warner 1976: 654)

Doch scheint die Medientheorie immerhin insoweit sprachorientiert konstituiert zu sein, als ihre allgemeinste Grundlage die linguistische Unterscheidung von Code und Botschaft (die auf Jacobson und Halle zurückgeht) sein soll (vgl. Lidz 1981: 44). Wie aber der Code-Begriff zu verstehen ist, wird von Parsons auf linguistischer Ebene nicht mehr konkretisiert. Er verwendet ihn abwechselnd im grammatisch-syntaktischen Sinn als System von Regeln zur Transformation und Kombination von Symbolen (vgl. Parsons 1968b: 470 und 465 mit explizitem Bezug auf Chomsky's Transformationsgrammatik) und im semantischen Sinn als lexikalisches System, das den Symbolen ihre Bedeutung als Gegenstandsbezug zuordnet (vgl. Parsons 1980b: 140; ebenso Jensen/Naumann 1980: 11). Baum setzt dann im Code-Begriff Syntax und Semantik in eins, indem er ihn als Regelsatz definiert, der die Grundelemente von Bedeutung (meaning) und die zulässigen Symbolkombinationen festlegt (vgl. 1976b: 538).

Sobald der Code-Begriff jedoch medientheoretisch konkretisiert wird, wird er nicht länger linguistisch gefaßt; Code ist dann, wie sich aus seinen Bestandteilen Wertprinzip, Koordinationsstandard und dem aus kulturellen oder legalen Institutionen bestehenden operativen Code unschwer ersehen läßt, ein Standard, der Systemrationalität und legitimen (nicht korrekten) Symbolgebrauch aufeinander abstimmt. Innerhalb der Medientheorie spielt deshalb die Bedeutung des Symbols und ihr Ursprung keine Rolle mehr. Dem semiotischen Dreieck aus Ausdruck (Symbol) - Gegenstand - Bedeutung wird die Spitze genommen (vgl. u. a. Schönrich 1981: 117ff.), indem die Bedeutung als Zeichenrelation zwischen Symbol und Gegenstand (das heißt: intrinsic satisfier) aufgefaßt wird (vgl. Parsons 1980b: 140; Cartwright/Warner 1976: 654), also auf eine eindimensionale, informationstheoretisch erfaßbare Zuordnungsbeziehung reduziert wird ${ }^{14}$. Durch diese Reduktion werden Sinnbegriff und Bedeutungsbegriff tautologisch (und damit obsolet), die Beziehung zwischen Symbol und Gegenstand wird mit dem Verhältnis von Geld zu konsummierbaren Gütern zur Deckung gebracht (vgl. Cartwright/Warner 1976: 654) und so für monetäre Interpretationen anschlußfähig gemacht.

Ferner macht es die eindimensionale Relation von Symbol und Gegenstand möglich, den pragmatischen Aspekt der Linguistik zu vernachlässigen: Parsons beachtet nicht, daß im Falle sprachlicher Kommunikation nicht die semantischen Symbole für intrinsic satisfiers stehen und mithin handlungsmotivierend wirken; daß vielmehr erst ihre pragmatische Einbettung zu konkretem Handeln motiviert: Bei einer ,Warnung vor dem Hunde' (Parsons' Lieblingsbeispiel) motiviert nicht der ,Hund", sondern die Warnung zur Flucht. ,Warnung' besitzt jedoch kein empirisches Signifikat, das als intrinsic satisfier aufgefaßt werden könnte, sondern weist den ganzen Sprechakt als exerzitive Äußerung aus (vgl. Austin 1979: 173).

Der Begriff der Sprache markiert bei Parsons nicht mehr und nicht weniger als ein Defizit: die eminente Bedeutung der Sprache wird zwar in programmatischen Erklärungen behauptet, jedoch nie systematisch erklärt - und von Parsons' Nachfolgern wieder dementiert ${ }^{15}$ (vgl. Jensen 1983: 56; Willke 1982: 122).

${ }^{14}$ Die informationstheoretische Reduktion des Sinnbegriffs durch die Gleichsetzung von Bedeutung mit einer einfachen Zeichenrelation ist tendenziell schon darin angelegt, daß Parsons sich damit zufrieden gibt, ,meaning' als Beziehung eines Symbols auf ein Objekt zu bestimmen. Eine genauere Bestimmung des Bedeutungsbegriffs (vgl. u. a. Dummett 1975 und 1977) hätte ergeben, daß sogar in dem Fall, der der Zeichenrelation auf den ersten Blick am ähnlichsten sieht, bei genauerem Hinsehen von einer ,einfachen' Beziehung Signifikans - Signifikat keine Rede sein kann: Eigennamen scheinen nur direkt auf Objekte bezogen zu sein; ihre Bedeutung zu verstehen, ist jedoch nicht ohne ein Hinzuziehen sortaler Prädikate möglich. „Wir brauchen nur an Freges Beispiel ,Nausikaa“ zu denken. ,Nausikaa' hat einen Sinn, der zum Teil durch den sortalen Ausdruck ,Mädchen" wiedergegeben wird. Ohne diesen Sinn könnte man nicht wissen, was die mutmaßliche Referenz von ,Nausikaa' sein soll wenn es überhaupt eine gibt." (Runggaldier 1985: 99)

${ }^{15}$ Außer von Lidz (1981). Auch Baums Beitrag (1976b) läuft auf eine eher sprachfreie Kommunikationstheorie hinaus. 


\section{Exkurs zu einer vermeintlichen Analogie zwischen den Medien und dem genetischen Code}

Jensen möchte, unter Berufung auf Parsons' Spätwerk, neben Geld und Sprache noch ein drittes paradigmatisches Grundmodell etablieren: den genetischen Code (vgl. 1984: 156ff.). Er unterscheidet zwei Funktionskomplexe, in denen Medien eine zentrale Rolle spielen: die Steuerung von Tausch- und Interaktionsprozessen und den ,raum/ zeitlichen Transfer kultureller Musterbildung' (vgl. 1984: 145). In Hinblick auf den postulierten Transfer-Aspekt der Medien sieht Jensen eine sprechende ,Analogie ${ }^{16}$ zum genetischen Code. Diese Analogisierung der symbolisch generalisierten Kommunikationsmedien mit dem genetischen Code ist jedoch nur um den Preis einer Entdifferenzierung der Parsons'schen Theorie möglich; sie widerspricht aber auch expliziten Äußerungen Parsons' zu möglichen Analogien zwischen ,Gegenständen'von Biologie und Action Theory.

Indem Jensen den Medien sowohl die Steuerung von Tausch- und Interaktionsprozessen als auch den Transfer kultureller Musterbildung zuordnet, vernachlässigt er Parsons' Differenzierung zwischen den symbolisch generalisierten Medien und den Mechanismen der Interpenetration. Die Übertragung kultureller Muster ist immer ein Fall von Interpenetration, sie kann aus strukturellen (und theoriebautechnischen) Gründen nicht von Medien übernommen werden. Luhmann hat diese Differenzierung schon 1978 gegenüber Jensen (1978) angemahnt: „Das Medienkonzept ist so gebaut, daß jedes Teilsystem seine Medien eigenständig codiert. Medien-Codes sind gerade nicht bloße Einrichtung des Transfers kultureller Wertmuster in die Teilsysteme; sie sind Codes für die Autonomisierung der Teilsysteme aus Anlaß der durch Differenzierung notwendig werdenden Tauschbeziehungen." (1978: 302)

Interpenetration bedeutet Vermittlung bestimmter Strukturen, die jedoch die Prozesse des interpenetrierten Systems nicht deterministisch bestimmen.

Parsons hat nun in der Tat seit den siebziger Jahren verstärkt Gewicht auf Parallelentwicklungen zwischen Biologie und struktur-funktionalistischer Action Theory gelegt, einer Parallelentwicklung,

${ }^{16}$ Diese Analogiebeziehung wird nur behauptet, nicht nachgewiesen (vgl. hierzu Weingartner 1979); dadurch aber hat sie, streng genommen, nur noch den Status einer Metapher (s. u. v. a. Brülisauer 1982).

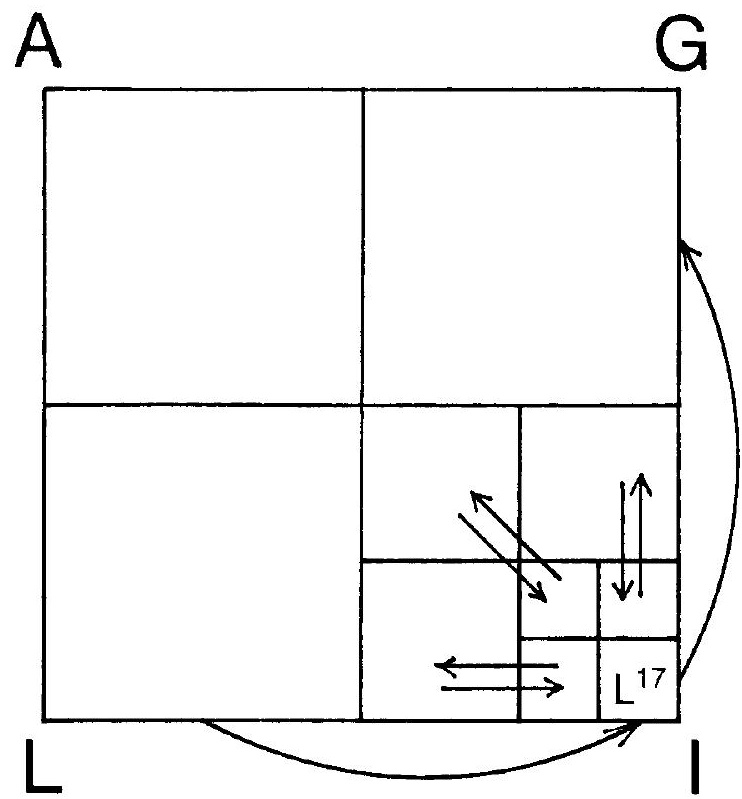

Abbildung 3 Das Verhältnis von Interpenetration und Medien (vgl. Luhmann 1978: 300).

die er in Analogien zwischen den jeweiligen Gegenständen verankert und insgesamt in den ,metaphysischen" Strukturen der conditio humana begründet sah, also in den Strukturen, die er in seinem kantianischen Spätwerk (vgl. Parsons 1978) zu analysieren versuchte. Diese Analogien hat Parsons sehr detailliert verfolgt (vgl. u. a. 1975a und 1982). Legt man seine Äußerungen zugrunde, kann man eine direkte Parallele oder Analogie zwischen genetischem Code und symbolisch generalisierten Medien definitiv ausschließen; die Analogien, die Parsons zieht, stützten auch die These von der konstitutiven Differenz zwischen Interpenetration und Medien. Analog verhalten sich Medien und genetischer Code allenfalls in Bezug auf den kybernetischen Informationsbegriff; beides sind Steuerungsinstanzen. Da der Informationsbegriff aber nicht ihr genus proximus ist, sondern im Gegenteil einer der obersten (und damit leeren) Begriffe in Parsons' Begriffs-Pyramidik, ist auch die bezüglich des Informationsbegriffs mögliche Analogie nichtssagend.

17 Bei Luhmann: I. Luhmann übernimmt hier die Veränderung, die Gould (1976: 471, 473) in der Anordnung des AGIL-Schemas vorgenommen hat und tauscht $\mathrm{L}$ Komponente und I-Komponente aus. 
Die Analogien, die Parsons sieht, lassen sich drei Ebenen zuordnen (Parsons 1982: 56f.; vgl. a. Rossi 1983: 188):

- ,Genotyp, unter den die biologische Spezies wie das Kultursystem fallen;

- ,Phänotyp', worunter das biologische Individuum wie die konkreten Subsysteme des Sozialsystems gehören und

- ,Vermittlungsagenturen', die die Verbindung zwischen Genotyp und Phänotyp herstellen; hierzu gehören die Interpenetrationsmechanismen und die Proteinbiosynthese.

Diese drei Ebenen lassen sich wie folgt schematisch darstellen:

Analog nennt Parsons nur Artbegriffe, die unter dasselbe genus proximus fallen:

"The patterns of culture are analogous to the genetic heritage of a species. This way of looking at it means drawing a careful distinction between the cultural and the social system, which is parallel to that drawn by biologists between germ plasm on the hand, somatoplasm on the other - or genotyp and phenotyp." (1975a: 76)

„It (. . .) seems clear that (. . ) the hormones are very closely analogons to action media. (. . .) The genes (. . .) are not anatomical parts of the phenotypical organism, nor are they physiological processes. They constitute a quite different order of factors." (1982: 58; andere Analogien sind Enzyme oder neurale Prozesse, vgl. 1975a: 79)

Von einer Analogie zwischen genetischem Code und symbolisch generalisierten Medien bei Parsons kann also keine Rede sein. Allenfalls könnte man

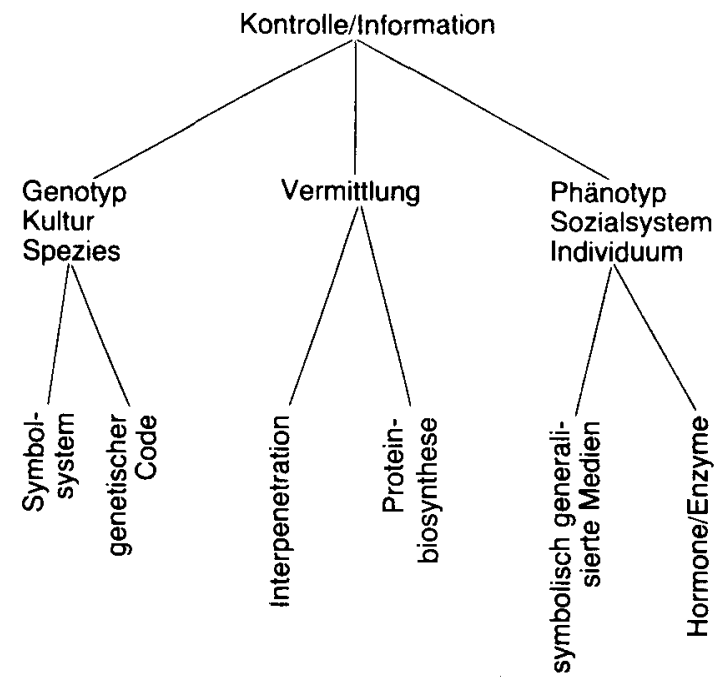

Abbildung 4 Das Verhältnis der Analogien zwischen Biologie und Action Theory. von einer funktionalen Analogie zwischen dem Code der Medien und dem genetischen Code sehen, müßte die Analogie dann aber durch Parallelisierung von biologischem Individuum und message-Ereignis kompletieren - die Künstlichkeit der Anordnung ist nicht zu übersehen.

\section{Die Schwierigkeiten der Geldanalogie bei der Anwendung auf die gesellschaftliche Ebene}

In evolutionärer Perspektive nimmt Parsons an, daß schon in primitiven Gesellschaften die Medien des allgemeinen Handlungssystems, also ,definition of situation', ,affect', ,performance capacity und ,intelligence' ausdifferenziert sind (vgl. Baum 1976b: 536). Erst wenn sich das Sozialsystem weiter differenziert, also erst in funktional differenzierten Gesellschaften, kann es zu einer komplementären Ausbildung der symbolisch generalisierten Medien Geld, Macht, Einfluß und Wertbindung kommen (vgl. Parsons 1968b: 471). Jedes Medium symbolisiert dabei eine allgemeine Kapazität; die Summe dieser Kapazitäten repräsentiert das Funktionieren des übergeordneten Systems.

- Geld symbolisiert die gesellschaftliche Kapazität der Verfügung über nützliche Güter und Dienstleistungen.

- Macht symbolisiert die gesellschaftliche Kapazität der Durchsetzung bindender Entscheidungen in Bezug auf kollektive Ziele.

- Einfluß symbolisiert die Kapazität, Konsens mit anderen Mitgliedern einer assoziativen Gemeinschaft zu erzielen, ohne eine vollständige Begründung mit sämtlichen relevanten Informationen geben zu müssen.

- Wertbindung symbolisiert die Kapazität, Werte auf der Ebene des Kollektivs zu implementieren (Jensen/Naumann 1980: 84).

Nun fällt auf, daß zwar einerseits in den Grundlagen der Theorie das linguistische Modell stillschweigend eliminiert worden ist, andererseits auf der Ebene der konkreten Anwendung des Konzepts auf das Sozialsystem das Geldmedium genausowenig zum Modell taugt.

Das Geldmedium besteht charakteristischerweise aus einem einzigen Symbol: der Währungseinheit, das durch einen numerischen ,Quantor' konkretisiert wird. Das Symbol kann natürlich in einer Vielzahl von Verwendungsformen auftauchen: als Hartgeld, Notengeld oder als Buch- bzw. Giralgeld - das Symbol, die quantifizierte Währungseinheit, 
bleibt mit sich identisch und als identische auch identifizierbar ${ }^{18}$. Sie ist es, die zirkuliert.

In zwei wesentlichen Punkten schert das Geldmedium aus der Medienfamilie aus, so da $\beta$ es fraglich wird, ob Parsons' „growing conviction that money could not be an isolated phenomenon but must be a member of a family of media" (zitiert nach Cartwright/Warner 1976: 652) haltbar ist: Diese Punkte sind die Skalierbarkeit und die Identifizierbarkeit der Geldsymbole ${ }^{19}$.

(1) Für die übrigen Medien des Sozialsystems lassen sich keine Symbole angeben, die der sich durchhaltenden Identifizierbarkeit der Geldsymbole auch nur annähernd entsprechen. Macht kann in einer Vielzahl von Formen auftreten: als Wahlakt (,A vote may be very little ,power' but it is power as a dollar is money.") (zit. nach Cartwright/ Warner 1976: 642)), als politische Erklärung, als legale Entscheidung (vgl. Baum 1976a: 463), als ,diskrete Mannigfaltigkeit von Machtsymbolen, die von Uniformen und Herrschaftsemblemen bis zum Amtssiegel und zur Unterschrift eines Zeichnungsberechtigten reichen" (Habermas 1981b: 86) - von Zirkulation kann hier nur noch in metaphorischem Sinne die Rede sein. Diese ,Machtformen können zwar als Analogie zu den verschiedenen Geldformen gedacht werden, jedoch läßt sich kein identisches Symbol ausmachen, das in allen Formen auftritt, also die Identität von Macht trotz und bei Überführbarkeit der Machtformen ähnlich ermöglicht, wie es die Identität der numerisch konkretisierten Währungseinheit gestatt, einen Scheck gegen Banknoten und diese gegen Hartgeld einzuwechseln. ${ }^{20}$

Das Medium Einfluß hat sich noch die größte Sprachnähe bewahrt. Sein Modus, also die Form der Medienverwendung, wird als ,Überredung' bestimmt. Gerade deshalb verbietet es sich aber auch, spezifische ,Einfluß'Symbole als Konstituentia der Botschaft (message) zu postulieren, da ,Überredung' nur auf der Ebene der Pragmatik identifiziert werden kann. Es ist kein Zufall, daß gerade für das Medium Einfluß keine Konkretisierungsvorschläge in Bezug auf die Symbolisierung existieren.

${ }^{18}$ Diese Identifizierbarkeit des Symbols verhehlen Jensen/Naumann (1980: 83), wenn sie gegen die Gleichsetzung des Geldes mit seinen physischen Repräsentationen den Vorwurf einer fallacy of misplaced concretness erheben.

${ }^{19}$ Für weitere Verstöße gegen die Geldanalogie s. a. Habermas (1981b: 87).
Für das Medium Wertbindung existieren zwei höchst heterogene Vorschläge in Bezug darauf, was hier als Symbol identifiziert werden könnte.

Baum schlägt als Symbolkategorie eine Klasse von ,rhetorical symbols“ vor: „They cover a wide variety, ranging from slogans of ,motherhood", ,fatherland' and ,socialism' over more composite forms such as national anthems to ,concretizations such as monuments signifying crucial collective moral tests." (1976a: 465)

- Jensen und Naumann dagegen sehen in Zeugnissen und Notenspiegeln als Commitment-Symbolen eine Parallele zur Währungseinheit als Geldsymbol (1980: 88).

Daß derart heterogene Vorschläge möglich und annähernd gleich plausibel sind, kann als Indiz dafür gewertet werden, daß der Begriff des Commitment-Symbols eine Leerformel darstellt.

Insgesamt besteht eine entscheidende Differenz zwischen Geldsymbol und den übrigen Symbolen der anderen Medien in Hinblick auf die Zeichenfunktion für intrinsic satisfiers beziehungsweise gesellschaftliche Kapazitäten: Das Geldmedium symbolisiert die Input/Output-Ströme zwischen der Wirtschaft und den übrigen Subsystemen des Sozialsystems, während die anderen Medien: Macht, Einfluß und Wertbindung durch die entsprechenden Input/Output-Ströme symbolisiert werden.

(2) Nicht viel besser als um die Identifizierbarkeit ist es um die Meßbarkeit und Skalierbarkeit der anderen Medien bestellt:

Geld kann offensichtlich mit einer Intervallskala erfaßt werden, (Baum zufolge [1976a: 463] sogar mit einer Rationalskala). Schon beim Medium Macht müssen hinsichtlich der Skalierbarkeit Abstriche gemacht werden: es kann, so Baum, nur mit einer Ordinalskala erfaßt werden. Und auch das dürfte nur durch Bezugnahme auf das im ope-

20 Jensen/Naumann meinen, das Problem mangelnder Identifizierbarkeit lösen zu können, indem sie das Defizit auf das Geldmedium übertragen: Sie argumentieren, daß die Währungseinheit nur eine manipulierbare Repräsentationseinheit sei, die das Symbol Geld symbolisiere (1980: 86). Jede andere Interpretation müsse das Geld mit seinen physischen Ausprägungen gleichsetzen, also eine fallacy of misplaced concretness begehen. Wenn aber das Geldsymbol überhaupt nicht existiert, auch nicht als Symbol, dann muß das Symbol (die Währungseinheit), das das nicht existente Symbol (das Geld) repräsentiert, selbst das Symbol sein. Was nichts anderes heißen kann, als daß Währungseinheit und Geldsymbol identisch sind. 
rativen Code verankerte Hierarchiemoment möglich sein. Wenn nicht durch klar umrissene Regeln der Weisungsbefugnis festgelegt ist, wer wem befehlen kann, wer Macht , ausgeben' darf, muß die Skalierung auf Nominalskalenniveau ermäßigt werden (zum Beispiel bei ungeklärter Kompetenzlage). - $\mathrm{Ob}$ dann aber noch von geldanaloger Meßbarkeit die Rede sein kann, ist fraglich. Parsons löst das Problem dieser Differenz zwischen Geld und Macht, indem er diesmal das Geldmedium so reduziert, daß die Analogie aufrechterhalten werden kann. Die lineare Meßbarkeit des Geldes verdankt sich, so Parsons, arbiträren empirischen Faktoren und hat mit den Grundlagen der Medientheorie selbst nichts zu tun (vgl. Cartwright/Warner 1976: 657 Anm. 22). Diese Unterschätzung der Skalierbarkeit des Geldes hängt damit zusammen, daß bei aller am Geldmechanismus orientierten Konstruktion der Medientheorie eine ausgearbeitete Preistheorie fehlt. Die Medientheorie reifiziert gegen alle Beteuerung das Geldmedium, da sie nicht beachtet, daß zur Preisbildung ein beliebiges Gut als Bezugseinheit, als numéraire (Walras) gewählt werden kann, das dann als Rechenmittel, als Geld dient. Der Preis stellt dann nichts anderes als die Austauschrelation der Güter untereinander dar (vgl. u. a. Ott 1975: 115).

Mag einer Skalierbarkeit des Machtmediums noch Plausibilität zugestanden werden - bei den Medien Einfluß und Wertbindung wird es prinzipiell fraglich ob von einer Skalierbarkeit auf der MessageEbene sinnvoll gesprochen werden kann. Zwar mag man auch von einem Mehr-oder-weniger an Einfluß sprechen können; die faktische Einflußnahme hängt jedoch nicht von Quantität oder Intensität der Symbole ab, sie verdankt sich vielmehr der Einstellung gegenüber der Person, die versucht, Einfluß auszuüben.

Zwar waren auf der Ebene der Grundlagen die Bruchstellen der Medientheorie noch unauffällig; sobald jedoch die Theorie einer Konkretisierung auf der Ebene des Sozialsystems unterworfen wird, zeigt es sich an den Ad-hoc-Argumentationen, die dann notwendig werden, daß es sich um ein inkonsistentes Konzept handelt: Geld kann nicht als Sprache aufgefaßt werden, Macht, Einfluß und Wertbindung können nicht zur Währung umgemünzt werden. Es war die These dieser Arbeit, daß sich diese Inkonsistenz der Medientheorie der Konkurrenz zwischen geldorientierter und sprachorientierter Konstruktion verdankt.

\section{Literatur}

Austin, J. L., 1979: Zur Theorie der Sprechakte. Stuttgart: Reclam.

Baum, R. C., 1976a: Introduction. S. 448-469 in: J. J. Loubser/R. C. Baum/A. Effrat/V. M. Lidz (Hrsg.), Explorations in General Theory in Social Science. Essays in Honour of Talcott Parsons. New York: The Free Press.

Baum, R. C., 1976b: Communication and Media. S. 533-556 in: J. J. Loubser/R. C. Baum/A. Effrat/V. M. Lidz (Hrsg.), Explorations in General Theory in Social Science. Essays in Honour of Talcott Parsons. New York: The Free Press.

Baum, R. C., 1976c: On Societal Media Dynamics. S. 579-608 in: J. J. Loubser/R. C. Baum/A. Effrat/V. M. Lidz (Hrsg.), Explorations in General Theory in Social Science. Essays in Honour of Talcott Parsons. New York: The Free Press.

Brülisauer, B., 1982: Der Erkenntniswert von metaphorischen Aussagen. Studia Philosophica 41: 177-199

Cartwright, B. C./Warner, R. S., 1976: The Medium Is Not the Message. S. 639-660 in: J. J. Loubser/R. C. Baum/A. Effrat/V. M. Lidz (Hrsg.), Explorations in General Theory in Social Science. Essays in Honour of Talcott Parsons. New York: The Free Press.

Coleman, J. S., 1963: Comment on ,On the Concept of Influence'. The Public Opinion Quarterly 27: 63-82.

Dummett, M., 1975: What Is a Theory of Meaning? S. 97-138 in: S. Guttenplan (Hrsg.), Mind and Language. London: Clarendon.

Dummett, M., 1977: What Is a Theory of Meaning? (II) S. 67-137 in: G. Evans/J. McDowell (Hrsg.), Truth and Meaning. Essays in Semantics. Oxford: Clarendon.

Ehrlicher, W., 1975: Geldtheorie. S. $352-420$ in: W. Ehrlicher/I. Esenwein-Rothe/H. Jürgensen/K. Rose, (Hrsg.), Kompendium der Volkswirtschaftslehre. Göttingen: Vandenhoeck.

Gould, M., 1976: Systems Analysis, Macrosociology, and the Generalized Media of Social Action. S. 470-506 in: J. J. Loubser/R. C. Baum/A. Effrat/V. M. Lidz (Hrsg.), Explorations in General Theory in Social Science. Essays in Honour of Talcott Parsons. New York: The Free Press.

Grünberger, J., 1981: Die Perfektion des Mitglieds. Berlin: Duncker \& Humblot.

Habermas, J., 1981a: Talcott Parsons - Probleme der Theoriekonstruktion. S. 28-48 in: J. Matthes (Hrsg.), Lebenswelt und soziale Probleme. Verhandlungen des 20. Dt. Soziologentages 1980. Frankfurt: Campus.

Habermas, J., 1981b: Handlung und System - Bemerkungen zu Parsons' Medientheorie. S. 68-105 in: W. Schluchter (Hrsg.), Verhalten, Handeln und System. Frankfurt: Suhrkamp.

Habermas, J., 1981c: Theorie des kommunikativen Handelns. Bd. 2: Zur Kritik der funktionalistischen Vernunft. Frankfurt: Suhrkamp. 
Jensen, S., 1978: Interpenetration - Zum Verhältnis personaler und sozialer Systeme? Zeitschrift für Soziologie 7: 116-129

Jensen, S., 1980: Einleitung. S. 7-56 in: S. Jensen (Hrsg.), Zur Theorie der sozialen Interaktionsmedien. Opladen: Westdeutscher Verlag.

Jensen, S./Naumann, J., 1980: Commitments: Medienkomponente einer ökonomischen Kulturtheorie? Zeitschrift für Soziologie 9: 79-99.

Jensen, S., 1983: Systemtheorie. Stuttgart: Kohlhammer. Jensen, S., 1984: Aspekte der Medientheorie: Welche Funktion haben Medien in Handlungssystemen? Zeitschrift für Soziologie 13: 145-164.

Lidz, V. M., 1981: Einleitung. S. 7-79 in: J. J. Loubser/ R. C. Baum/A. Effrat/V. M. Lidz (Hrsg.), Allgemeine Handlungstheorie. Frankfurt: Suhrkamp.

Lipp, W., 1979: Kulturtypen, kulturelle Symbole, Handlungswelt. Zur Plurivalenz von Kultur. Kölner Zeitschrift für Soziologie und Sozialpsychologie 31: 450-484.

Loubser, J. J., 1981: Handlung und Erlebnis. S. 328-394 in: J. J. Loubser/R. C. Baum/A. Effrat/V. M. Lidz (Hrsg.), Allgemeine Handlungstheorie. Frankfurt: Suhrkamp.

Luhmann, N., 1968: Zweckbegriff und Systemrationalität. Frankfurt: Suhrkamp.

Luhmann, N., 1976: Generalized Media and the Problem of Contingency. S. 507-532 in: J. J. Loubser/R. C. Baum/A. Effrat/V. M. Lidz (Hrsg.), Explorations in General Theory in Social Science. Essays in Honour of Talcott Parsons. New York: The Free Press.

Luhmann, N., 1977: Interpenetration - Zum Verhältnis personaler und sozialer Systeme. Zeitschrift für Soziologie 6: 62-76.

Luhmann, N., 1978: Interpenetration bei Parsons. Zeitschrift für Soziologie 7: 299-302.

Luhmann, N., 1980a: Talcott Parsons - Zur Zukunft eines Theorieprogramms. Zeitschrift für Soziologie 9: 5-17

Luhmann, N., 1980b: Gesellschaftsstruktur und semantische Tradition. S. 9-71 in: N. Luhmann, GesellschaftsStruktur und Semantik. Bd. 1, Frankfurt: Suhrkamp.

Luhmann, N., 1984: Die Wirtschaft der Gesellschaft als autopoietisches System. Zeitschrift für Soziologie 13: 308-327.

Münch, R., 1980a: Über Parsons zu Weber: Von der Theorie der Rationalisierung zur Theorie der Interpenetration. Zeitschrift für Soziologie 9: 18-3.

Münch, R., 1980b: Talcott Parsons und die Theorie des Handelns II. Soziale Welt 31: 3-47.

Ott, A. E., 1975: Preistheorie. S. 114-182 in: W. Ehrlicher/I. Esenwein-Rothe/H. Jürgensen/K. Rose (Hrsg.), Kompendium der Volkswirtschaftslehre, Göttingen: Vandenhoeck.

Parsons, T./Smelser, N., 1956: Economy and Society. London: Routledge.

Parsons, T., 1968a: Social Interaction. S. 429-440 in: D. Sills (Hrsg.), International Encyclopedia of the Social Sciences. Vol. 7. New York.

Parsons, T., 1968b: Social Systems. S. $458-473$ in: D.
Sills (Hrsg.), International Encyclopedia of the Social Sciences. Vol. 15. New York

Parsons, T., 1970: Some Problems of General Theory in Sociology. S. 27-68 in: J. C. McKinney/E. A. Tiryakian (Hrsg.), Theoretical Sociology; Perspectives and Development. New York: Appelton.

Parsons, T., 1975a: The Present Status of „StructuralFunctional" Theory in Sociology. S. 67-83 in: L. A. Coser (Hrsg.), The Idea of Social Structure. Papers in Honor of Robert K. Merton. New York: Harcourt.

Parsons, T., 1975b: Gesellschaften. Frankfurt: Suhrkamp.

Parsons, T., 1978: A Paradigm of the Human Condition. S. 352-433 in: T. Parsons, Action Theory and the Human Condition. New York: The Free Press.

Parsons, T., 1980a: Über den Begriff der Macht. S. 57-137 in: S. Jensen (Hrsg.), Zur Theorie der sozialen Interaktionsmedien. Opladen: Westdeutscher Verlag.

Parsons, T., 1980b: Über den Begriff ,Einfluß' S. 138-182 in: S. Jensen (Hrsg.), Zur Theorie der sozialen Interaktionsmedien. Opladen: Westdeutscher Verlag.

Parsons, T., 1980c: Über den Begriff ,Commitments'. S. 183-228 in: S. Jensen (Hrsg.), Zur Theorie der sozialen Interaktionsmedien. Opladen: Westdeutscher Verlag.

Parsons, T., 1980d: Sozialstruktur und die symbolischen Tauschmedien. S. 229-259 in: S. Jensen (Hrsg.), Zur Theorie der sozialen Interaktionsmedien. Opladen: Westdeutscher Verlag.

Parsons, T., 1982: Action, Symbols, and Cybernetic Control. S. 49-65 in: I. Rossi (Hrsg.), Structural Sociology. Theoretical Perspektives and Substantive Analyses. New York.

Rossi, I., 1983: From the Sociology of Symbols to the Sociology of Signs. Toward a Dialectical Sociology. New York: Columbia University Press.

Runggaldier, E., 1985: Zeichen und Bezeichnetes. Sprachphilosophische Untersuchungen zum Problem der Referenz. Berlin: Dunker \& Humblot.

Schluchter, W., 1981: Gesellschaft und Kultur - Überlegungen zu einer Theorie institutioneller Differenzierung. S. 106-149 in: W. Schluchter (Hrsg.), Verhalten, Handeln und System. Frankfurt: Suhrkamp.

Schönrich, G., 1981: Kategorien und transzendentale Argumentation. Frankfurt: Suhrkamp.

Shwayder, D. S., 1965: The Stratification of Behaviour. A System of Definitions Propounded and Defended. London: Routledge.

Stichweh, R., 1980: Rationalität bei Parsons. Zeitschrift für Soziologie 9: 54-78.

Turner, T. S., 1968: Parsons' Concept of Generalized Media of Social Interaction and its Relevance for Social Anthropology. Sociological Inquiry 38: $121-134$

Weingartner, P., 1979: Analogy among Systems. Dialectica 33: 355-378.

Willke, H., 1982: Systemtheorie. Stuttgart-New York: Fischer. 